\title{
Generalized Open Sets of Minkowski Space
}

\author{
Soley Ersoy, Merve Bilgin, And İBrahim İnCe
}

\begin{abstract}
We consider the Euclidean topology and $s$-topology on $n$-dimensional Minkowski space and investigate the interior and closure of the space cone and perforated space cone with respect to these topologies. In these regards, we determine that whether the space cone and perforated space cone are generalized open set or not. Moreover, we compare the generalized Euclidean topologies with the generalized $s$-topologies.
\end{abstract}

\section{INTRODUCTION}

In 1963, Zeeman explained that considering Euclidean topology, which is locally homogenous, on Minkowski space is not good enough since the null cone separates the spacelike vectors from the timelike ones in this space [25]. Zeeman suggested a new topology on Minkowski space $M$ and introduced the finest topology on 4-dimensional Minkowski space that induces 1-dimensional Euclidean topology on any timelike line and 3-dimensional Euclidean topology on any spacelike hyperplane with their homeomorphisms groups generated by inhomogenous Lorentz group, translations and dilatations $[25,26]$. These topologies were called, respectively, time topology and space topology by [16]. Also, Nanda defined $t$-topology and $s$-topology which are, respectively, weaker than the Zeemans' time and space topology $[16,17]$. The order topology (Whiston called it Zeeman-order topology [24]) and $A$-topology on the Minkowski space were studied in $[18,19]$, respectively. Dossena obtained the results that the Zeemans' topology as the finest topology on $n$-dimensional Minkowski space is separable, Hausdorff, non-normal, non-locally compact, non-Lindeloff and the first countable and moreover, he investigated 2-dimensional case [12]. The topological properties of $t$-topology and $s$-topology were studied and the characterizations of the compact sets of Minkowski space with these topologies were obtained by $[2,3]$.

2010 Mathematics Subject Classification. 53B30, 54A05.

Key words and phrases. Generalized topology, Minkowski space. 
To the best of authors' knowledge generalized topology of the Minkowski space is not studied yet. In the last century the theory of generalized topology has been widely studied in the literature. The classes of subsets of a topological space which are more and less nearly open sets have been represented throughout the past few decades in the field of topology. In 1963, Levine defined semi-open sets and gave some properties of the class of these open-like sets, [14]. Then a large number of studies have been devoted to investigate the properties of $\alpha$-open, $\beta$-open, semi-open, pre-open, regularopen and $b$-open sets $[14,20,15,1,4,22,23,5]$. Let $(X, \tau)$ be a topological space and $A$ be a subset of $X$. The closure and the interior of $A$ are denoted by $c_{\tau}(A)$ and $\operatorname{int}_{\tau}(A)$, respectively. The subset $A$ of $(X, \tau)$ is called a regular-open set, if $A=\operatorname{int}_{\tau}\left(c_{\tau}(A)\right)$ [22]. The finite union of regular open sets is said to be $\pi$-open in $(X, \tau)$. The family of all regular open sets is denoted by $R O(X)$, and $R O(X) \subset \tau$. Also, a subset $A$ of $(X, \tau)$ is called $\alpha$-open [20] (resp., $\beta$-open [1, 4], semi-open [14], pre-open[15], and $b$-open [5]) set if $A \subset \operatorname{int}_{\tau}\left(c_{\tau}\left(\operatorname{int}_{\tau}(A)\right)\right)$ (resp., $A \subset c_{\tau}\left(\operatorname{int}_{\tau}\left(c l_{\tau}(A)\right)\right), A \subset$ $c l_{\tau}\left(\operatorname{int}_{\tau}(A)\right), A \subset \operatorname{int}_{\tau}\left(c l_{\tau}(A)\right)$, and $\left.A \subset c_{\tau}\left(\operatorname{int}_{\tau}(A)\right) \cup \operatorname{int}_{\tau}\left(c l_{\tau}(A)\right)\right)$. The family of all $\alpha$-open (resp., $\beta$-open, semi-open, pre-open, and $b$-open) sets in $(X, \tau)$ is denoted by $\alpha O(X)(\beta O(X), S O(X), P O(X)$, and $B O(X))$ and there are the relations $\tau \subset \alpha O(X) \subset S O(X) \subset B O(X) \subset \beta O(X)$ and $\tau \subset \alpha O(X) \subset P O(X)[8,13]$.

Some common properties of these well-known sets were considered and more general definitions were given by Császár in 1997, [6]. The remarkable class of studies on generalized open sets was given by Császár, [7, 9, 10, 11]. The collection $\zeta$ of subsets of a non-empty $X$ was called generalized topology by Császár such that, $\emptyset \in \zeta$, and arbitrary unions of elements of $\zeta$ belong to $\zeta$. The collection of the generalized open sets defined in [6] constitutes a generalized topology. A set $X$ with a generalized topology $\zeta$ was called as a generalized topological space, [7].

In these regards, we study generalized open subsets of Minkowski space endowed with the Euclidean topology and $s$-topology, respectively.

\section{Preliminaries}

The Lorentzian inner product of the vectors $x=\left(x_{0}, x_{1}, \ldots, x_{n-1}\right)$ and $y=$ $\left(y_{0}, y_{1}, \ldots, y_{n-1}\right)$ is defined to be $g(x, y)=-x_{0} y_{0}+\sum_{i=1}^{n-1} x_{i} y_{i}$. The real vector space $\mathbb{R}^{n}$ provided with the Lorentzian inner product $g$, which is symmetric, non-degenerate bilinear form, is called $n$-dimensional Minkowski space and denoted by $M$. Since $g$ is an indefinite form, recall that $x \in M$ can have three Lorentzian casual character as; it can be spacelike if $g(x, x)>0$ or $x=0$, timelike if $g(x, x)<0$, and null (lightlike) if $g(x, x)=0$ and $x \neq 0$, [21]. The group of linear operators $T$ on $M$ which leaves Lorentzian inner product $g$ invariant as $g(x, x)=g(T(x), T(x))$ for all $x \in M$ is called Lorentz 
group. The sets

$$
\begin{aligned}
& C^{S}(x)=\{y \in M: y=x \text { or } g(y-x, y-x)>0\} \\
& C^{L}(x)=\{y \in M: g(y-x, y-x)=0\}, \quad \text { and } \\
& C^{T}(x)=\{y \in M: y=x \text { or } g(y-x, y-x)<0\}
\end{aligned}
$$

are space cone, null (lightlike) cone, and time cone of $x \in M$, respectively. The Euclidean topology on $n$-dimensional Minkowski space $M$ is the topology generated by the basis $B=\left\{N_{\varepsilon}^{E}(x): \varepsilon>0, x \in M\right\}$ which will be called $e$-topology. $M$ with the Euclidean topology will be denoted by $M^{E}$ and the elements of Euclidean topology on Minkowski space will be called $e$-open sets.

The $s$-topology on the $n$-dimensional Minkowski space $M$ is defined by specifying the local base of neighborhoods at each point $x \in M$ given by the collection $N(x)=\left\{N_{\varepsilon}^{S}(x): \varepsilon>0\right\}$, where $N_{\varepsilon}^{S}(x)=N_{\varepsilon}^{E}(x) \cap C^{S}(x)$. We call $N_{\varepsilon}^{S}(x)$ the $s$-neighborhhood of radius $\varepsilon$. $M$ endowed with $s$-topology is denoted by $M^{S}$ and the elements of $s$-topology on the Minkowski space will be called $s$-open sets.

Hence $U \subseteq M$ is $s$-open with respect to $s$-topology if and only if there exists some $N_{\varepsilon}^{S}(x)$ such that $N_{\varepsilon}^{S}(x) \subseteq U$ for each $x \in U$.

Let us recall characters of cones in Minkowski space with respect to $s$-topology and $e$-topology from the following Lemmas given by [3].

Lemma 2.1. Let $M$ be $n$-dimensional Minkowski space and $x \in M$. Then $C^{T}(x)-\{x\}$ and $C^{S}(x)-\{x\}$ are open in $M^{E}$ and $C^{L}(x)$ is closed in $M^{E}$ $[3]$.

Lemma 2.2. Let $M$ be $n$-dimensional Minkowski space and $x \in M . N_{\varepsilon}^{S}(x)$, $\varepsilon>0$, is open in $M^{S}[3]$.

Lemma 2.3. Let $M$ be $n$-dimensional Minkowski space and $x \in M$. Then

i. $C^{S}(x)$ is not open in $M^{E}$,

ii. $C^{S}(x)$ is open in $M^{S}[3]$.

Lemma 2.4. The s-topology on $M$ is strictly finer than the Euclidean topology on $M[3]$.

Similar definitions and theorems are also available for $t$-topology on the $n$-dimensional Minkowski space $M$.

\section{Some Generalized Open Sets of Minkowski Space}

In this section, we introduce the interior and closure of some important subsets of Minkowski space with respect to the $s$-topology and $e$-topology. Then, we investigate that, whether these sets are generalized open set or not with respect to these topologies. 
Lemma 3.1. Let $M$ be $n$-dimensional Minkowski space and $x \in M$. Then $C^{S}(x) \cup C^{L}(x)$ is closed in $M^{E}$.

Proof. $C^{T}(x)-\{x\}$ is open in $M^{E}$. So $M-\left(C^{T}(x)-\{x\}\right)$ is closed in $M^{E}$.

$$
M-\left(C^{T}(x)-\{x\}\right)=\left(\left(C^{S}(x) \cup C^{L}(x)\right)-\{x\}\right) \cup\{x\}=C^{S}(x) \cup C^{L}(x)
$$
completes the proof.

Lemma 3.2. Let $M^{E}$ be $n$-dimensional Minkowski space endowed with $e$-topology and $x \in M$. Then

i. $\operatorname{int}_{e}\left(C^{S}(x)\right)=C^{S}(x)-\{x\}$,

ii. $\operatorname{cl}_{e}\left(C^{S}(x)\right)=C^{S}(x) \cup C^{L}(x)$,

iii. int $_{e}\left(C^{S}(x)-\{x\}\right)=C^{S}(x)-\{x\}$,

iv. $\operatorname{cl}_{e}\left(C^{S}(x)-\{x\}\right)=C^{S}(x) \cup C^{L}(x)$,

v. $\operatorname{int}_{e}\left(C^{S}(x) \cup C^{L}(x)\right)=C^{S}(x)-\{x\}$,

vi. $\operatorname{cl}_{e}\left(C^{S}(x) \cup C^{L}(x)\right)=C^{S}(x) \cup C^{L}(x)$.

Proof. i. From the Lemma 2.1 and Lemma $2.3(i)$ the proof is trivial. ii. $C^{S}(x) \cup C^{L}(x)$ is an $e$-closed set in $M^{E}$ and $C^{S}(x) \subset\left(C^{S}(x) \cup\right.$ $\left.C^{L}(x)\right)$. Since $\mathrm{cl}_{e}\left(C^{S}(x)\right)$ is the smallest $e$-closed set containing $C^{S}(x), c_{e}\left(C^{S}(x)\right)$ is smaller than $C^{S}(x) \cup C^{L}(x)$, that is $c l_{e}\left(C^{S}(x)\right) \subset\left(C^{S}(x) \cup C^{L}(x)\right)$.

Conversely, let $y \in\left(C^{S}(x) \cup C^{L}(x)\right)$. Then $y \in C^{S}(x)$ or $y \in$ $C^{L}(x)$. If $y \in C^{S}(x)$ then it is obvious that $y \in \operatorname{cl}_{e}\left(C^{S}(x)\right)$. On the other case, if $y \in C^{L}(x)$ then there exists $\exists z \in N_{\varepsilon}^{E}(y)$ for $\forall \varepsilon>0$, such that $z \in C^{S}(x)$. Thus for $\forall \varepsilon>0, N_{\varepsilon}^{E}(y) \cap C^{S}(x) \neq \emptyset$. This gives us $y \in c l_{e}\left(C^{S}(x)\right)$ and $\left(C^{S}(x) \cup C^{L}(x)\right) \subset c l_{e}\left(C^{S}(x)\right)$. This proves the assertion.

iii. Since $C^{S}(x)-\{x\}$ is an $e$-open set, this case is obvious.

iv. $\left(C^{S}(x)-\{x\}\right) \subset C^{S}(x)$. This requires $c l_{e}\left(C^{S}(x)-\{x\}\right) \subset c l_{e}\left(C^{S}(x)\right)$.

From $(i i)$ it is easily seen that $c l_{e}\left(C^{S}(x)-\{x\}\right) \subset\left(C^{S}(x) \cup C^{L}(x)\right)$. Conversely, let $y \in\left(C^{S}(x) \cup C^{L}(x)\right)$. From $(i i) y \in \operatorname{cl}_{e}\left(C^{S}(x)\right)$. If $y \neq x$ then $y \in \operatorname{cl}_{e}\left(C^{S}(x)-\{x\}\right)$ or if $y=x$, then for $\forall \varepsilon>0$, $N_{\varepsilon}^{E}(y) \cap\left(C^{S}(x)-\{x\}\right) \neq \emptyset$, that is, $y \in c_{e}\left(C^{S}(x)-\{x\}\right)$. In either case $\left(C^{S}(x) \cup C^{L}(x)\right) \subset c l_{e}\left(C^{S}(x)-\{x\}\right)$. Finally, we obtain $c l_{e}\left(C^{S}(x)-\{x\}\right)=C^{S}(x) \cup C^{L}(x)$.

v. $C^{S}(x) \subset\left(C^{S}(x) \cup C^{L}(x)\right)$ requires int ${ }_{e}\left(C^{S}(x)\right) \subset \operatorname{int}_{e}\left(C^{S}(x) \cup C^{L}(x)\right)$ and from $(i)\left(C^{S}(x)-\{x\}\right) \subset \operatorname{int}_{e}\left(C^{S}(x) \cup C^{L}(x)\right)$. Conversely, let $y \in \operatorname{int}_{e}\left(C^{S}(x) \cup C^{L}(x)\right)$. Since $\operatorname{int}_{e}\left(C^{S}(x) \cup C^{L}(x)\right) \subset\left(C^{S}(x) \cup\right.$ $\left.C^{L}(x)\right), y \in\left(C^{S}(x) \cup C^{L}(x)\right)$. Hence either $y \in\left(C^{L}(x)-\{x\}\right)$, $y=x$ or $y \in\left(C^{S}(x)-\{x\}\right)$. But it is easy to see that $y$ is not an interior point when $y \in\left(C^{L}(x)-\{x\}\right)$ or $y=x$ since there isn't any Euclidean neighborhood $N_{\delta}^{E}(y)$ with radius $\delta$ such that $N_{\delta}^{E}(y) \subset$ 
$\left(C^{S}(x) \cup C^{L}(x)\right)$. Thus, $\operatorname{int}_{e}\left(C^{S}(x) \cup C^{L}(x)\right) \subset\left(C^{S}(x)-\{x\}\right)$. This completes the proof.

vi. This assertion is obvious since $C^{S}(x) \cup C^{L}(x)$ is an $e$-closed set in $M^{E}$.

Lemma 3.3. Let $M^{S}$ be $n$-dimensional Minkowski space endowed with $s$-topology and $x \in M$. Then

i. $\operatorname{int}_{s}\left(C^{S}(x)\right)=C^{S}(x)$,

ii. $c l_{s}\left(C^{S}(x)\right)=C^{S}(x) \cup C^{L}(x)$,

iii. $\operatorname{int}_{s}\left(C^{S}(x)-\{x\}\right)=C^{S}(x)-\{x\}$,

iv. $\operatorname{cl}_{s}\left(C^{S}(x)-\{x\}\right)=C^{S}(x) \cup C^{L}(x)$,

v. $\operatorname{int}_{s}\left(C^{S}(x) \cup C^{L}(x)\right)=C^{S}(x)$,

vi. $\operatorname{cl}_{s}\left(C^{S}(x) \cup C^{L}(x)\right)=C^{S}(x) \cup C^{L}(x)$.

Proof. By considering the definitions of $s$-open and $s$-closed sets in $M^{S}$ the proofs can be done by similar a manner of proofs of Lemma 3.2.

Lemma 3.4. Let $M^{E}$ be $n$-dimensional Minkowski space endowed with $e$-topology. Then the space cone $C^{S}(x)$ is

i. $\sigma-e-o p e n$,

ii. $b-e-o p e n$,

iii. $\beta-e$-open set in $M^{E}$,

although $C^{S}(x)$ is not

iv. $\alpha-e-o p e n$,

v. pre $-e-$ open,

vi. $r-e-$ open set in $M^{E}$.

Proof. i. From the Lemma $3.2(i)$ and $(i v)$, one can see $C^{S}(x) \subset$ $c l_{e}\left(\operatorname{int}_{e}\left(C^{S}(x)\right)\right)$ and hence $C^{S}(x)$ is $\sigma-e$-open set in $M^{E}$.

ii. By considering the above assertion and Lemma $3.2(i i)$ and $(v)$, we get $C^{S}(x) \subset c_{e}\left(\operatorname{int}_{e}\left(C^{S}(x)\right)\right) \cup \operatorname{int}_{e}\left(c_{e}\left(C^{S}(x)\right)\right)$. Hence $C^{S}(x)$ is $b-e$-open set in $M^{E}$.

iii. From the Lemma $3.2(i i),(v)$ and $(i v), C^{S}(x) \subset c_{e}\left(\operatorname{int}_{e}\left(c_{e}\left(C^{S}(x)\right)\right)\right)$ is obtained and hence $C^{S}(x)$ is $\beta-e$-open set in $M^{E}$.

iv. From the Lemma $3.2(i),(i v)$ and $(v), C^{S}(x) \not \subset \operatorname{int}_{e}\left(c_{e}\left(\operatorname{int}_{e}\left(C^{S}(x)\right)\right)\right)$ that is, $C^{S}(x)$ is not $\alpha-e$-open set in $M^{E}$.

v. From the Lemma $3.2(i i)$ and $(v), C^{S}(x) \not \subset$ int $_{e}\left(c_{e}\left(C^{S}(x)\right)\right)$ and this means that $C^{S}(x)$ is not pre-e-open set in $M^{E}$.

vi. From the Lemma $3.2(i i)$ and $(v)$, int $_{e}\left(c l_{e}\left(C^{S}(x)\right)\right) \neq C^{S}(x)$ that is, $C^{S}(x)$ is not $r-e$-open set in $M^{E}$.

Lemma 3.5. Let $M^{S}$ be $n$-dimensional Minkowski space endowed with $s$-topology. Then the space cone $C^{S}(x)$ is $\alpha-s$-open, $\beta-s$-open, $\sigma-$ $s$-open, pre-s-open, $b-s-o p e n$, and $r-s$-open set in $M^{S}$. 
Proof. $C^{S}(x)$ is a $s$-open set in $M^{S}$ from the Lemma 2.3. Since $s \subset$ $\alpha O\left(M^{S}\right) \subset S O\left(M^{S}\right) \subset B O\left(M^{S}\right) \subset \beta O\left(M^{S}\right)$ and $s \subset \alpha O\left(M^{S}\right) \subset$ $P O\left(M^{S}\right)$ it is easily seen that $C^{S}(x)$ is $\alpha-s$-open, $\beta-s$-open, $\sigma-s$-open, pre-s-open, and $b-s$-open set in $M^{S}$. But we need to show whether $C^{S}(x)$ is $r-s$-open set or not in $M^{S}$ since $R O\left(M^{S}\right) \subset s$. From the Lemma 3.3 (ii) and (v) one can see $\operatorname{int}_{s}\left(c_{s}\left(C^{S}(x)\right)\right)=C^{S}(x)$ and hence $C^{S}(x)$ is $r-s$-open set in $M^{S}$.

Lemma 3.6. Let $M^{E}$ be n-dimensional Minkowski space endowed with $e$-topology. Then the perforated space cone $C^{S}(x)-\{x\}$ is $\alpha-e-$-open, $\beta-e-o p e n, \sigma-e-o p e n$, pre-e-open, and $b-e-$ open and $r-e-$ open set in $M^{E}$.

Proof. $C^{S}(x)-\{x\}$ is an $e$-open set in $M^{E}$ from the Lemma 2.1. Also, $e \subset \alpha O\left(M^{E}\right) \subset S O\left(M^{E}\right) \subset B O\left(M^{E}\right) \subset \beta O\left(M^{E}\right)$ and $e \subset \alpha O\left(M^{E}\right) \subset$ $P O\left(M^{E}\right)$. So $C^{S}(x)-\{x\}$ is $\alpha-e$-open, $\beta-e$-open, $\sigma-e-$ open, pre-e-open, and $b-e$-open set in $M^{E}$. Since $R O\left(M^{E}\right) \subset e$ we need to prove whether $C^{S}(x)-\{x\}$ is $r-e$-open set or not in $M^{E}$. From the Lemma 3.2 (iv) and (v) $\operatorname{int}_{e}\left(c_{e}\left(C^{S}(x)-\{x\}\right)\right)=C^{S}(x)-\{x\}$ is seen and hence $C^{S}(x)-\{x\}$ is $r-e$-open set in $M^{E}$.

Lemma 3.7. Let $M^{S}$ be $n$-dimensional Minkowski space endowed with $s$-topology. Then the perforated space cone $C^{S}(x)-\{x\}$ is $\alpha-s$-open, $\beta-s$-open, $\sigma-s$-open, pre-s-open, and $b-s$-open set in $M^{S}$. But $C^{S}(x)-\{x\}$ is not $r-s$-open set in $M^{S}$.

Proof. $C^{S}(x)-\{x\}$ is $e$-open set in $M^{E}$ from the Lemma 2.1 and also $C^{S}(x)-\{x\}$ is $s$-open set in $M^{S}$ from Lemma 2.4. From the relations $s \subset \alpha O\left(M^{S}\right) \subset S O\left(M^{S}\right) \subset B O\left(M^{S}\right) \subset \beta O\left(M^{S}\right)$ and $s \subset \alpha O\left(M^{S}\right) \subset$ $P O\left(M^{S}\right)$ we see that $C^{S}(x)-\{x\}$ is $\alpha-s$-open, $\beta-s$-open, $\sigma-s$-open, pre-s-open, and $b-s$-open set in $M^{S}$. Let us investigate whether $C^{S}(x)-$ $\{x\}$ is $r-s$-open set or not in $M^{S}$. From the Lemma 3.3 (iv) and (v) one can see $\operatorname{int}_{s}\left(\operatorname{cl}_{s}\left(C^{S}(x)-\{x\}\right)\right) \neq C^{S}(x)-\{x\}$ and hence $C^{S}(x)-\{x\}$ is not $r-s$-open set in $M^{S}$.

Lemma 3.8. If $G$ is any e-open set in $M^{E}$. Then $G$ is $\alpha, \sigma, b, \beta$, and pre-s-open set in $M^{S}$.

Proof. $G$ is arbitrary $e$-open set in $M^{E}$. From the Lemma 2.4, it follows that $G$ is $s$-open set in $M^{S}$. $G$ is an $\alpha, \sigma, b, \beta$, and pre-s-open set in $M^{S}$ since $s \subset \alpha O\left(M^{S}\right) \subset S O\left(M^{S}\right) \subset B O\left(M^{S}\right) \subset \beta O\left(M^{S}\right)$ and $s \subset$ $\alpha O\left(M^{S}\right) \subset P O\left(M^{S}\right)$.

Lemma 3.9. Let $M$ be $n$-dimensional Minkowski space. Then the collection of $\alpha-s$-open set $\alpha(s)$ is strictly finer than the collection of $\alpha-e-$ open sets $\alpha(e)$. 
Proof. Suppose that $G$ is arbitrary $\alpha-e-$ open set in $M^{E}$. Then $G \subset$ $\operatorname{int}_{e}\left(c_{e}\left(\operatorname{int}_{e}(G)\right)\right)$. From the Lemma 2.4, it follows that;

$$
\begin{aligned}
& \operatorname{int}_{e}(G) \subset \operatorname{int}_{s}(G) \\
\Rightarrow & c l_{e}\left(\operatorname{int}_{e}(G)\right) \supset c l_{s}\left(\operatorname{int}_{s}(G)\right) \\
\Rightarrow & \operatorname{int}_{e}\left(c l_{e}\left(\operatorname{int}_{e}(G)\right)\right) \subset \operatorname{int}_{s}\left(c_{s}\left(\operatorname{int}_{s}(G)\right)\right) \\
\Rightarrow & G \subset \operatorname{int}_{e}\left(c l_{e}\left(\operatorname{int}_{e}(G)\right)\right) \subset \operatorname{int}_{s}\left(c_{s}\left(\operatorname{int}_{s}(G)\right)\right) \\
\Rightarrow & G \subset \operatorname{int}_{s}\left(c l_{s}\left(\operatorname{int}_{s}(G)\right)\right) \\
\Rightarrow & G \text { is } \alpha-s-\text { open set in } M^{S} .
\end{aligned}
$$

Hence the collection of $\alpha-s$-open sets $\alpha O\left(M^{S}\right)$ is finer than the collection of $\alpha-e$-open sets $\alpha O\left(M^{E}\right)$. Also, from Lemma 3.4 and Lemma 3.5, the collection of $\alpha-s$-open sets $\alpha O\left(M^{S}\right)$ is strictly finer than the collection of $\alpha-e-$ open sets $\alpha O\left(M^{E}\right)$.

Lemma 3.10. Let $M$ be $n$-dimensional Minkowski space. Then the collection of pre-s-open sets $P O\left(M^{S}\right)$ is strictly finer than the collection of pre-e-open sets $P O\left(M^{E}\right)$.

Proof. Let $G$ be an arbitrary pre-e-open set in $M^{E}$. Then $G \subset \operatorname{int}_{e}\left(c l_{e}(G)\right)$. From the Lemma 2.4, it follows that;

$$
\begin{aligned}
& c l_{e}(G) \supset c l_{s}(G) \\
\Rightarrow & \operatorname{int}_{e}\left(c l_{e}(G)\right) \subset \operatorname{int}_{s}\left(c l_{s}(G)\right) \\
\Rightarrow & G \subset \operatorname{int}_{e}\left(c l_{e}(G)\right) \subset \operatorname{int}_{s}\left(c l_{s}(G)\right) \\
\Rightarrow & G \subset \operatorname{int}_{s}\left(c l_{s}(G)\right) \\
\Rightarrow & G \text { is pre }-s-\text { open set in } M^{E} .
\end{aligned}
$$

Hence the collection of pre-s- open sets $P O\left(M^{S}\right)$ is finer than the collection of pre-e- open sets $P O\left(M^{E}\right)$. Also, from the Lemma 3.4 and Lemma 3.5 , the collection of pre-s-open sets $P O\left(M^{S}\right)$ is strictly finer than the collection of pre- $e-$ open sets $P O\left(M^{E}\right)$.

Also, the collections of $\beta, \sigma, r, b-e$-open and $s$-open sets are not comparable.

\section{REFERENCES}

[1] M.E. Abd El-Monsef, S.N. El-Deeb and R.A. Mahmoud, $\beta$-open sets and $\beta$ continuous mapping, Bull. Fac. Sci. Assiut Univ. A, A12, (1983), no. 1, 77-90.

[2] G. Agrawal and S. Shrivastava, $t$-topology on the $n$-dimensional Minkowski space, J. Math. Phys., 50(5), (2009), Article ID 053515, 6 pages.

[3] G. Agrawal and S. Shrivastava, A study of non-Euclidean s-topology, ISRN Mathematical Physics, (2012), Article ID 896156, 11 pages.

[4] D. Andrijević, Semipreopen sets, Mat. Vesnik, 3, (1986), no. 1, 24-32. 
[5] D.Andrijević, On b-open sets, Mat. Vesnik, 48 (1996), 59-64.

[6] Á. Császár, Generalized open sets, Acta Math. Hungar., 75 (1997), no. 1-2, 65-87.

[7] Á. Császár, Generalized topology, generalized continuity, Acta Math. Hungar., 96 (2002), no. 4, 351-357.

[8] Á. Császár, Generalized open sets in generalized topologies, Acta Math. Hungar., 106 (2005), 53-66.

[9] Á. Császár, Generalized open sets and generalized topologies, Mat. Lapok (N.S.), 11 (2002/03), no. 1, 11-15 (2006).

[10] Á. Császár, Further remarks on the formula for $\gamma$-interior, Acta Math. Hungar., 113 (2006), no. 4, 325-332.

[11] Á. Császár, $\delta$ - and $\theta$-modifications of generalized topologies, Acta Math. Hungar., 120, (2008), no.3, 275-279, 54A05.

[12] G. Dossena, Some results on the Zeeman topology, J. Math. Phys., 48(11) (2007), Article ID 113507, 13 pages.

[13] E. Ekici, On weak structures due to Császár, Acta Math. Hungar., 134(4) (2012), 565-570.

[14] N. Levine, Semi-open sets and semi-continuity in topological spaces, Amer. Math. Monthly, 70 (1963), 36-41.

[15] A.S. Mashhour, M.E. Abd El-Monsef and S.N. El-Deep, On precontinuous and weak precontinuous mappings, Proc. Math. Phys. Soc. Egypt, 53 (1982), 47-53(1983).

[16] S. Nanda, Weaker versions of Zeeman's conjectures on topologies for Minkowski space, J. Math. Phys., 13 (1972), 12-15.

[17] S. Nanda, Topology for Minkowski space, J.Math.Phys., 12 (1971), 394-401.

[18] S. Nanda and H.K. Panda, Minkowski space with order topology is simply connected, Internat. J. Theoret. Phys., 12(6) (1975), 393-399.

[19] S. Nanda, A-topology for Minkowski Space, J. Austral. Math. Soc. Ser. B, 21 (1979), 53-64.

[20] O. Njästad, On some classes of nearly open sets, Pacific J. Math., 15 (1965), 961-970.

[21] B. O'Neill, Semi-Riemannian geometry with applications to relativity, Academic Press, New York (1983).

[22] M. H. Stone, Applications of the theory of boolean rings to general topology, Trans. Amer. Math. Soc., 41(1937), 357-381.

[23] N.V. Veličko, H-closed topological spaces, Mat. Sb. (N.S.), 70(112) (1966), 98-112.

[24] G.S. Whiston, The Zeeman-order topology on Minkowski space, Internat. J. Theoret. Phys., 6 (1972), 75-76.

[25] E.C. Zeeman, Causality implies the Lorentz group, J. Math. Phys., 5 (1964), 490-493.

[26] E.C. Zeeman, The topology of Minkowski space, Topology, 6 (1966), 161-170.

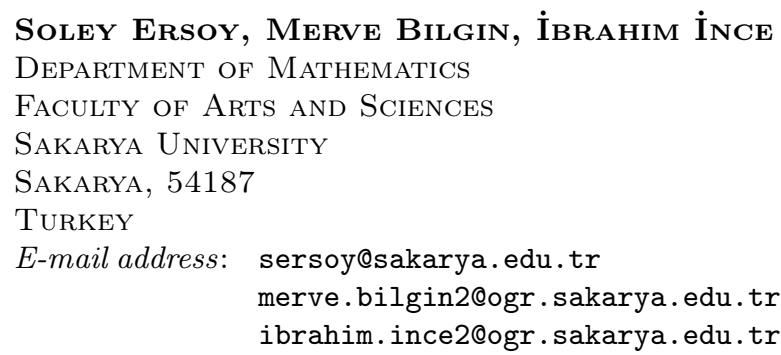

